\title{
Low Voltage Scanning Electron Microscopy - Current Status, Present Problems, and Future Solutions -*
}

\author{
David C. Joy ${ }^{\dagger}$ \\ Electron Beam Laboratory, Dept. of Materials Science and Engineering, \\ University of Tennessee, Knoxville, TN 37996-2200, USA, and \\ Center for NanoPhase Materials Science, Oak Ridge National Laboratory, Oak Ridge, TN, USA
}

(Received 25 October 2005; Accepted 15 February 2006; Published 16 April 2006)

\begin{abstract}
Low voltage scanning electron microscopy (LVSEM) has become the imaging technique of choice for many applications because it offers surface specific information, and both control of charging and a reduction of damage to labile specimens. However fundamental limitations such as the electron wavelength, lens aberrations, and gun brightness, severely limit the performance of the LVSEM, and in addition some capabilities such as microanalysis are difficult or impossible. New technologies, including aberration correction and brighter electron sources are now available, and novel spectroscopic approaches are in development. Together these advances could significantly enhance the performance of the LVSEM. The advent of ultra-high performance ion column imaging systems offers interesting competition to the LVSEM for both imaging and analysis. [DOI: 10.1380/ejssnt.2006.369]
\end{abstract}

Keywords: SEM; secondary electrons; ion beams; electron spectroscopy

\section{INTRODUCTION}

Low Voltage Scanning Electron Microscopes (LVSEMs) which operate at accelerating voltages below $1 \mathrm{kV}$ have now become the standard configuration for many purposes, supplanting the older, conventional, SEMs which operated at energies between 15 and $30 \mathrm{keV}$. This transformation has occurred because a low beam energy ensures that all signal forming interactions occur within a few nanometers of the surface. Operation at low energies can also, in many cases, minimize charging artifacts in insulators and semiconductors and restrict the physical extent - if not the magnitude - of beam induced damage. However, by comparison with a higher energy SEM the electron optical performance of low voltage instruments is severely compromised by two problems - the aberrations of the electron-optical column, and the limited gun brightness. In addition, the LVSEM lacks effective technologies for performing microanalysis. If the LVSEM is going to remain a key imaging and analytical tool for advanced research and technology then these limitations must be overcome. The purpose of this paper is to review the current status of low voltage imaging and to outline new solutions to the problems which now restrict performance.

\section{ELECTRON OPTICAL LIMITATIONS IN THE LVSEM}

The electron optical performance of a LVSEM is limited primarily as the result of two factors, lens aberrations and the relatively large wavelength of low energy electrons. Three major lens errors can be distinguished. Chromatic aberration is the focusing error which occurs because the

*This paper was presented at 5th International Symposium on Atomic Level Characterizations for New Materials and Devices (ALC05), Hawaii, USA, 4-9 December, 2005.

†Corresponding author: djoy@utk.edu electron beam is not mono-energetic. All electron sources produce a beam of electrons with a finite energy width $\Delta E$, which generally increases with the temperature of the source. Thus a thermionic emitter operating at 2800 $\mathrm{K}$ typically has $\Delta E \sim 2.5 \mathrm{eV}$ while a field emitter which runs at room temperature has $\Delta E \sim 0.3 \mathrm{eV}$ The minimum focused spot (disc of least confusion) diameter $d_{C h r}$ produced by the lens as a result of chromatic aberration is then

$$
d_{C h r}=C_{c} \alpha \frac{\Delta E}{E}
$$

where $E$ is the energy of the emitted electron, and $\alpha$ is the convergence angle of the focused beam. $C_{c}$ is the chromatic aberration coefficient of the lens, a parameter with units of length and whose magnitude is typically of the order of the working distance of the lens. As $E$ becomes smaller the significance of chromatic aberration becomes correspondingly greater.

The wavelength $\lambda$ of non-relativistic electrons is given to sufficient accuracy by the relation

$$
\lambda[\mathrm{nm}]=\frac{1.26}{E^{1 / 2}} .
$$

The diameter $d_{D i f f}$ of the diffraction limited spot into which these electrons can be focused is then

$$
d_{\text {Diff }}=0.61 \frac{\lambda}{\alpha}
$$

Finally, there is also spherical aberration which occurs because paraxial rays passing through a lens are brought to a focus at a point which is further away from the lens than peripheral rays. If the object (in this case the electron source) is of zero size then the diameter of the disc of least confusion $d_{S p h}$ is

$$
d_{S p h}=0.5 C_{s} \alpha^{3}
$$

where $C_{s}$ is the spherical aberration coefficient of the lens and is again to a first approximation equal in magnitude to the working distance. 


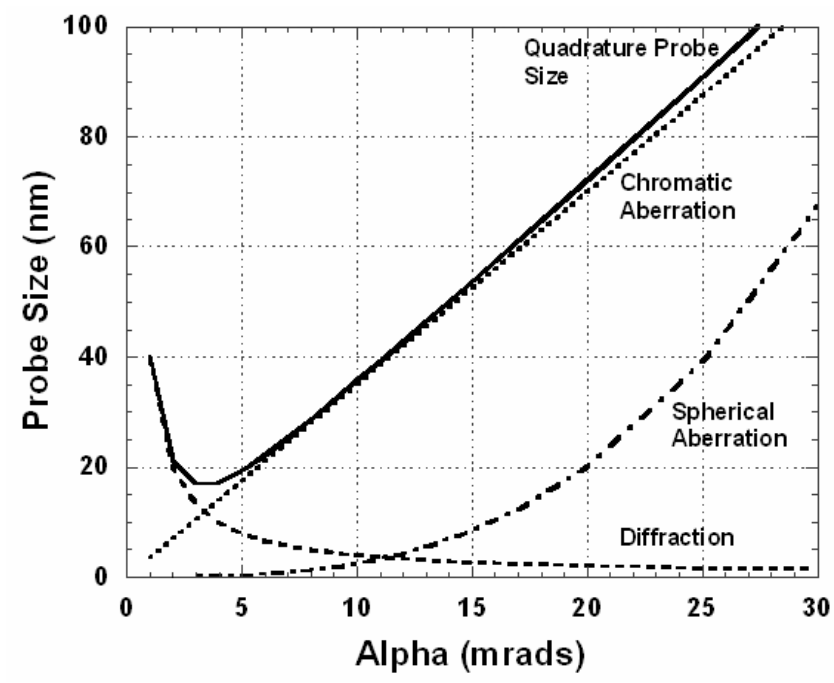

FIG. 1: The variation of chromatic and spherical aberrations, and diffraction, as a function of beam convergence angle in an uncorrected SEN operating at $1 \mathrm{keV}$ beam energy and their contributions to the probe size. The $C_{s}$ and $C_{c}$ aberration coefficients are taken to be $5 \mathrm{~mm}$, and the energy spread $\Delta E$ of the Schottky Emitter is $0.7 \mathrm{eV}$.

Figure 1 plots these contributions to the probe size of a modern LVSEM, assuming typical lens parameters for a working distance (WD) of $4 \mathrm{~mm}$ :- a $C_{s}$ value of $5 \mathrm{~mm}$, a $C_{c}$ value of $5 \mathrm{~mm}$ - a beam energy of $1 \mathrm{keV}$, and a $\Delta E$ spread of $0.7 \mathrm{eV}$ from a Schottky emitter. The effect of each of the different aberrations is shown separately together with the final probe size estimated by adding these contributions in quadrature. It is clear that, depending on the choice of $\alpha$, the final probe size is dominated by either diffraction or by chromatic aberration (together with a small contribution from spherical aberration). At still lower energies the contributions from both diffraction and chromatic aberrations would be higher and the probe size would become significantly larger. The optimum operating choice for $\alpha$ will be the largest angle possible (because the beam current increases as $\alpha^{2}$ ) which also simultaneously minimizes the diffraction and the aberration contributions. For the parameters chosen here, this occurs when $\alpha$ is 4 to 5 milli-radians (mrad) yielding a spot size of about $18 \mathrm{~nm}$. While this operating condition provides a relatively good resolution and a high depth of field because of the tiny numerical aperture, these benefits are achieved at the expense of strongly limiting the current $I_{B}$ in the beam, and hence degrading the signal to noise ratio of the image.

It must be admitted that the probe size predicted here is much larger than the resolution promised by most of the high performance LVSEMs that are currently available. This is partly because the lens parameters used here have been given values which are conservative compared to those which might be encountered, for example, in LVSEMs which use a cathode lens, or 'beam boost', arrangement to enhance performance. However, the 'resolution' values typically quoted for commercial instruments are invariably highly optimistic because they have been measured from images of samples which are not well cho- sen or characterized for such uses, and have been analyzed by methods which are not reliable or reproducible.

Enhancing LVSEM performance implies lowering the probe size while simultaneously increasing the beam current. Fortunately, recent developments - specifically the development of aberration correctors, and the advent of nano-tip field emitter sources - have now made such an improvement a possibility.

\section{ABERRATION CORRECTION}

Scherzer in 1937 [1] proved that all electron-optical lenses displayed positive-definite aberrations, but in a later paper [2] he showed that this result did not extend to lenses which were either non-cylindrically symmetric, or in which the electron energy or lens excitation varied with time. In the decades since Scherzer's papers there has thus been a large amount of work to develop devices which can exploit these exceptions and so reduce or eliminate the aberrations of conventional lenses. Currently two commercial systems with these capabilities, one from CEOS (Germany) and another from NION (USA), are now available $[3,4]$.

Both of these devices rely on a combination of 'multipole' magnetic lenses - i.e. lenses consisting of a finite number of discrete magnetic poles spaced around the beam axis - because this arrangement does not possess cylindrical symmetry and so satisfies one of Scherzer's conditions. By assembling several such multi-pole lenses together a corrector can then be made which reduces or cancels all of the lowest order aberrations of the objective lens [5]. The resultant compound lens now has effective aberrations coefficients which are determined by the excitations of the various elements in the multi-pole device, allowing both the $C_{c}$ and the $C_{s}$ aberration coefficients to be adjusted to be zero, or positive, or negative as desired. The ability to control the aberrations in this way naturally implies that some adjustment and alignment are required. Because of the large number of optical components involved (typically 48, but often 64 ), and because of the mutual interactions between them, manual adjustment is not practical and a fully computerized system must be used to set up and to subsequently monitor the performance. The complexity of this procedure and the computing power required to manage it during operation has been a factor in delaying the arrival of practical correction systems.

\section{THE RESULTS OF ABERRATION CORRECTION IN A LVSEM}

Figure 2 shows how the contributions of the various aberration components analyzed in Figure 1 behave, as a function of the convergence angle, if correction is applied. The parameters assumed here are the same as those Figure 1 but both $C_{c}$ and $C_{s}$ are assumed to have been effectively reduced to 50 micrometers. (Note that choosing to set both of these components to zero is not necessarily a useful option because higher order aberration coefficients, not eliminated by the corrector, then become dominant 


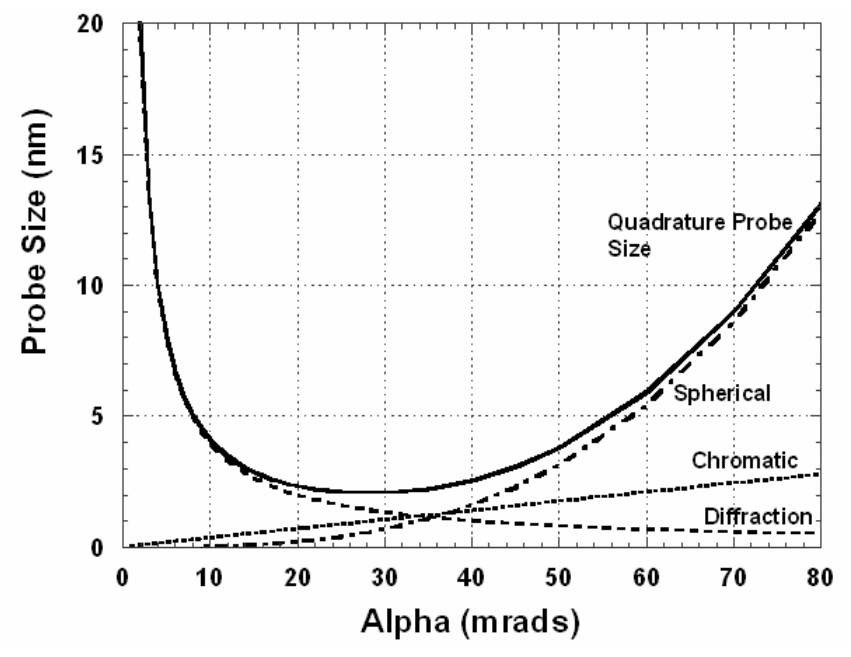

FIG. 2: As Figure 1, but in an aberration corrected SEM. The $C_{s}$ and $C_{c}$ aberration coefficients are now set to 50 micrometers.

and may result in unpredictable behavior). At convergence angles of between 1 to $10 \mathrm{mrad}$. the beam size is still limited, as before, by diffraction but $\alpha$ can now be increased to 30 or even 40 mrad. before the chromatic and spherical aberration contributions become large enough for the total probe size to begin to increase. As a result the achievable minimum probe size now falls to about 2.5 $\mathrm{nm}$ compared to its uncorrected value of $18 \mathrm{~nm}$, and this is achieved at an optimum value of $\alpha$ of around $35 \mathrm{mrad}$ as compared to the original value of 4 to $5 \mathrm{mrad}$. The effect of aberration correction is therefore to simultaneously reduce the probe size while increasing the current contained in the probe (which varies as $\alpha_{o p t}^{2}$ ) by a factor of more than $30 \times$, a combination which appears to satisfy the requirements for both better resolution and more current.

The first commercial example of an aberration corrected LVSEM, the JSM $7700 \mathrm{~F}$, is now available from JEOL [5], and comparable instruments from other manufacturers are promised. Thus far no detailed evaluation of the performance of any aberration corrected SEM has been made so it is too early to know if the predictions of enhanced performance are fully borne out in practice. Nevertheless it is certain that this development will revolutionize the LVSEM by enhancing all aspects of its performance. For example, in addition to the benefits listed earlier it is also worth noting that aberration correction also implies that the overall instrument performance is no longer determined by the working distance (WD) of the lens. This will make it possible to increase the distance between the specimen and the lens without penalizing performance. The space thus made available can then be used to place new, and more effective, detectors and spectrometers for image and data acquisition. In addition the intensity profile of the probe becomes closely Gaussian both at, and on either side, of focus although the depth of field is much reduced because of the enlarged numerical aperture of the corrected lens. On the debit side the additional cost of the correctors is considerable at $\$ 300 \mathrm{k}$ to $\$ 500 \mathrm{k}$, and requires other aspects of the instrumentation

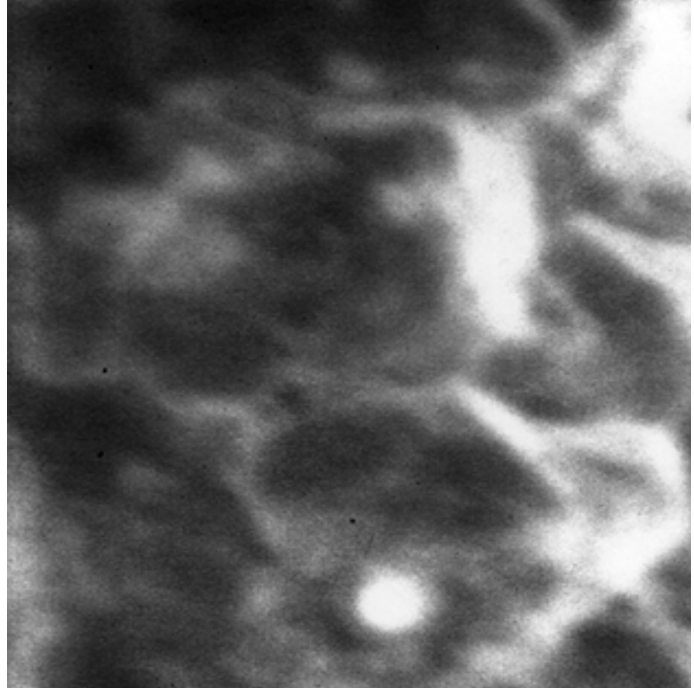

(a)

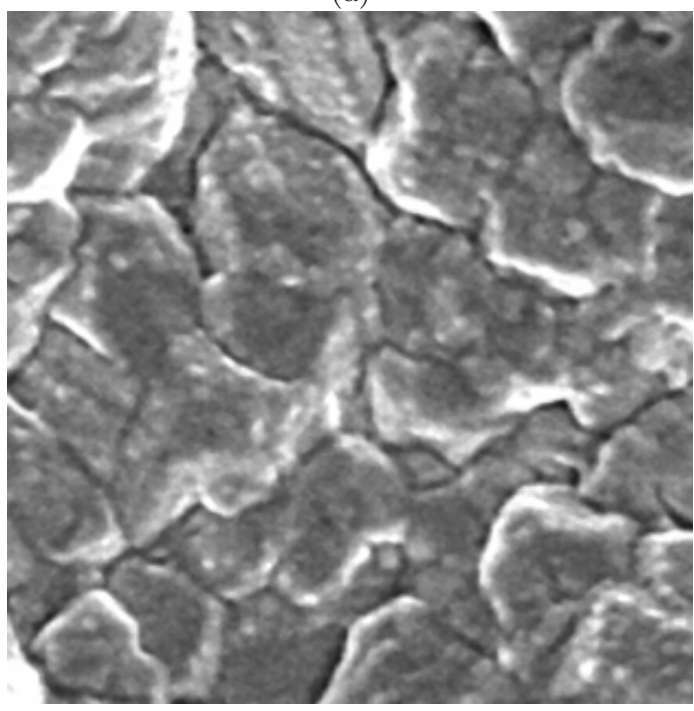

(b)

FIG. 3: A comparison of SE images from the same sample (a copper grid) in an SEM column operating at $0.9 \mathrm{keV}$ beam energy but equipped with (a) a conventional cold field emitter source and (b) a nano-tip cold field emitter. Field of view 2 micrometers. Unpublished data courtesy A. Vladar.

- such as the stability of lens and gun power supplies - to be upgraded. Finally their complexity will make routine operation more challenging as well as rewarding.

\section{HIGH PERFORMANCE ELECTRON EMITTERS}

A benefit of employing aberration corrected lenses is that the amount of current in the beam is increased by a factor of 25 to $30 \times$ as compared to that available from the same electron source but with uncorrected optics. While this is a welcome increase it is insufficient to ensure an adequate signal to noise ratio at low energies because, as shown by Langmuir's relation [6], the brightness of all electron sources falls linearly with the beam energy $E$. As a result a field emission gun operating at $500 \mathrm{eV}$ is 


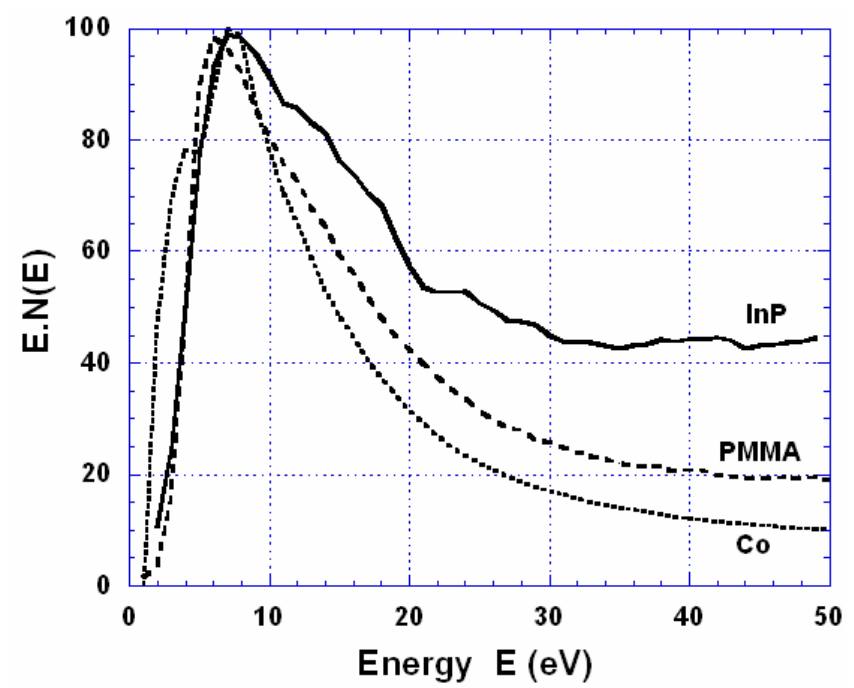

FIG. 4: Secondary electron spectra recorded at $1 \mathrm{keV}$ from a metal (cobalt), an insulator (PMMA photoresist) and a semiconductor (InP). To reduce the dynamic range of the data the spectra are plotted as $E \cdot N(E)$ vs. $E$, where $E$ is the kinetic energy of the secondary and $N(E)$ is the secondary emission at $E$ and the data has been normalized.

only about as bright as a thermionic gun at $30 \mathrm{keV}$. In the energy regime below $1 \mathrm{keV}$ the secondary electron yield $\delta$ is also falling as $E$ [7], so overall the SE signal drops as about $E^{2}$. Overcoming this constraint to performance requires new electron sources of much higher intrinsic brightness, and a practical example of this is the Nano-tip field emitter [8]. These are conventional field emitter tips which have been formed by special thermal and electrolytic processing so that the emission comes from a single atom at the tip. The reduced brightness (amps $/ \mathrm{cm}^{2} /$ steradian/volt) of such an emitter is about $100 \times$ higher that of a conventional field emitter, and the atomic source size minimizes the need for demagnifying optics so simplifying the optical design of the column (although putting a higher premium on mechanical rigidity and stability). Because the tip radius is less than a nanometer cleaning the tip by 'flashing' it is not possible, but the stability and reliability of operation have still been found to be satisfactory even in modest vacuum levels. Initial experimental results with such emitters [9] demonstrate (Figure 3) that useful improvements in resolution due to the reduction in source size and chromatic spread and overall image quality - from the enhanced beam current - are achieved even on a less than optimized electron - optical column. The improvement in brightness from a nano-tip will be especially important at ultra-low beam energies.

\section{MICROANALYSIS AT LOW ENERGIES}

The ability to combine chemical identification with imaging is such an important benefit of scanning microscopes that its absence on low voltage SEMs is a serious deficiency. The problem is that conventional electron fluoresced X-rays are of limited analytical value because the low incident beam energy cannot excite detectable lines from most of the elements of interest, and Auger spectroscopy is not usually an option because the vacuum environment of most SEMs is too dirty. However, for many of the industrial and research purposes for which the LVSEM is now employed (for example defect review in the semiconductor industry) the main details of sample chemistry are completely determined, so the requirement is not for a technique to identify unknown elements, but for one which can rapidly and reliably verify the chemistry of localized regions. At least two approaches are possible contenders.

\section{NEW APPROACHES TO MICRO-ANALYSIS IN THE LVSEM}

\section{A. Secondary Electron Spectroscopy}

One option is that of secondary electron spectroscopy. SE spectroscopy has not been actively pursued as an analytical method because of the assumption that in the relatively poor vacuum of a typical SEM the secondary spectrum would be representative only the surface layer of hydrocarbon and water vapor rather than of the details of the underlying substrate. However recent experimental work $[10,11]$ shows that the details of the portion of the $\mathrm{SE}$ spectrum below about $20 \mathrm{eV}$ (typically representing $70 \%$ or more of the total intensity) are essentially the same from both 'as received', and consequently contaminated, surfaces and from a surface cleaned in site by an ion beam in ultra-high vacuum. This is consistent with the fact that the inelastic mean free path for such low energy secondaries is large (10 to $30 \mathrm{~nm}$ ) and consequently their energy distribution is not significantly changed as they diffuse through a shallow surface layer of contaminant. As illustrated in Figure 4 the differences between the SE spectra from a metal, a semiconductor, and a polymer insulator, are readily evident and studies have confirmed that the profile of these spectra is reproducible when compared from different instruments, or after significant time intervals [11]. Software analysis of SE spectra employing software neural network procedures [11] has been found capable of distinguishing between such spectra with high reliability after suitable training. There is, at the moment, no spectrometer design optimized for SE operation but the widespread use in high performance SEMs of the pre-field of the objective lens together with an electrostatic field to form a Wien ("E $\times \mathbf{B}$ ") filter to selectively transmit electrons to a secondary detector indicates that the basic technology already exists.

\section{B. Time of Transit Spectroscopy}

The interaction of energetic electrons with a solid is usually treated as being an instantaneous event but, in reality, the time taken between the entry of an electron into a solid and the emergence of a backscattered or secondary electron is finite and a knowledge of timings associated with this transit could form the basis of a new spectroscopy for materials analysis [12]. Transit time spectra can be modeled by a Monte Carlo trajectory tracing 


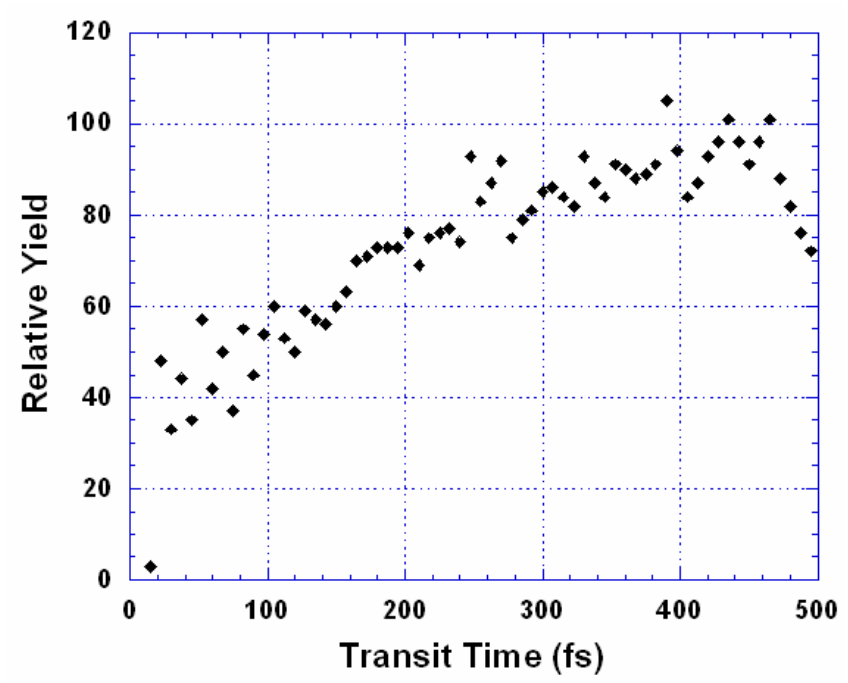

(a)

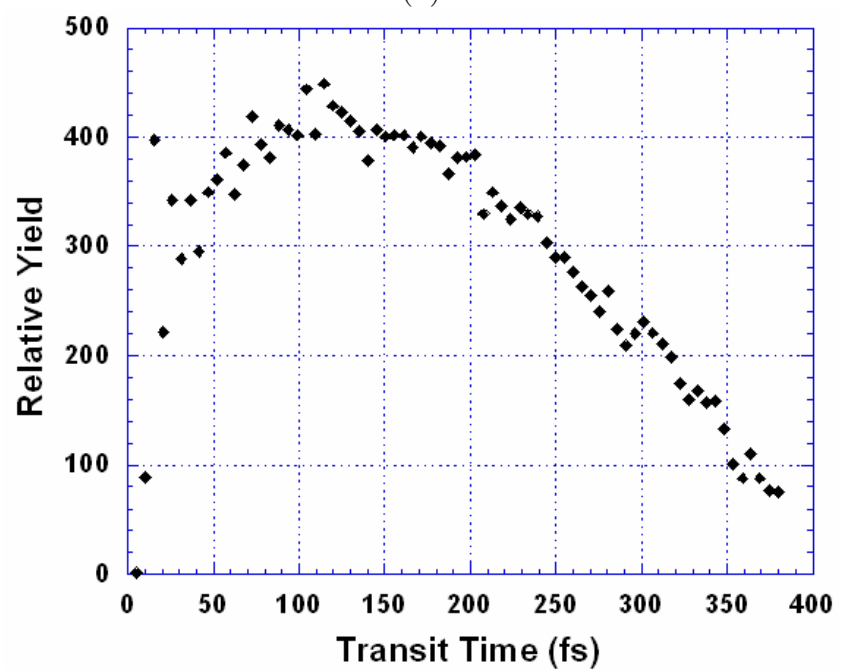

(b)

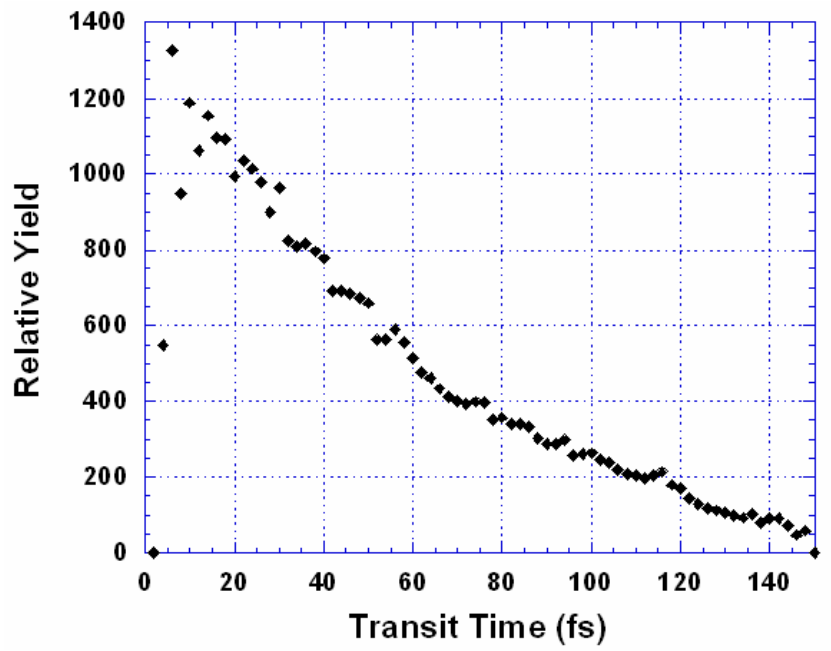

(c)

FIG. 5: Time of transit ("splash") spectra simulated by a Monte Carlo model for the emission of backscattered electrons from (a) carbon, (b) titanium, and (c) gold for $10 \mathrm{keV}$ incident electrons.

method since the position and the velocity of every electron is known at all times [13]. For example, with a beam

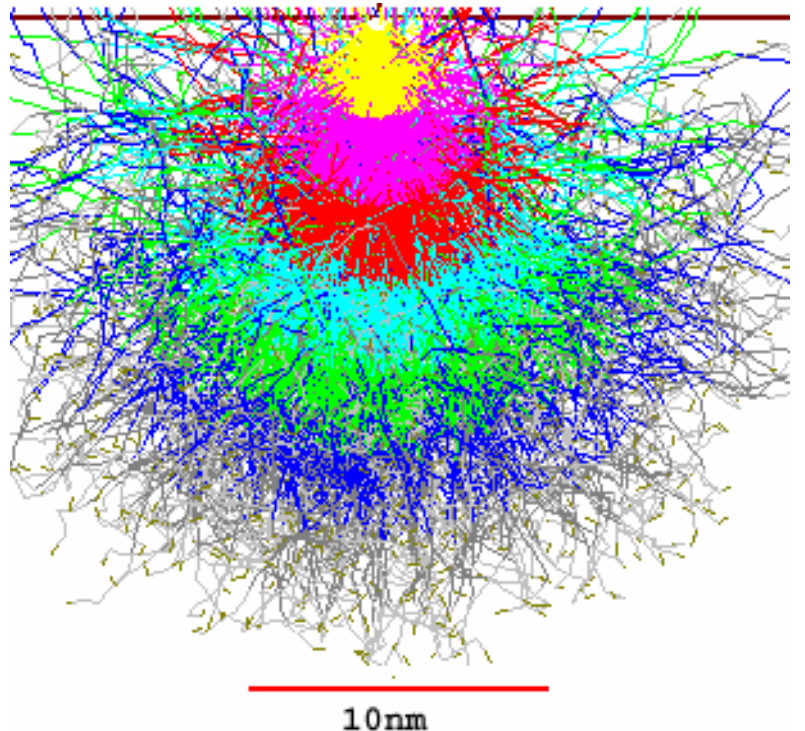

(a)

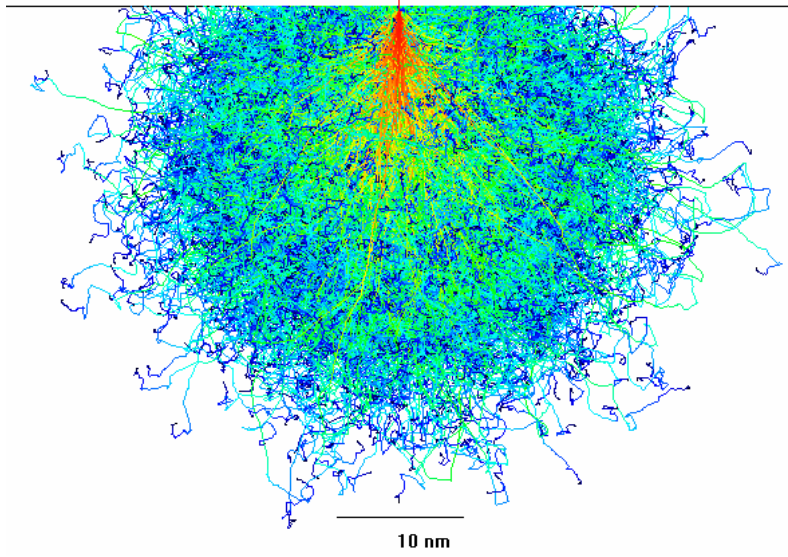

(b)

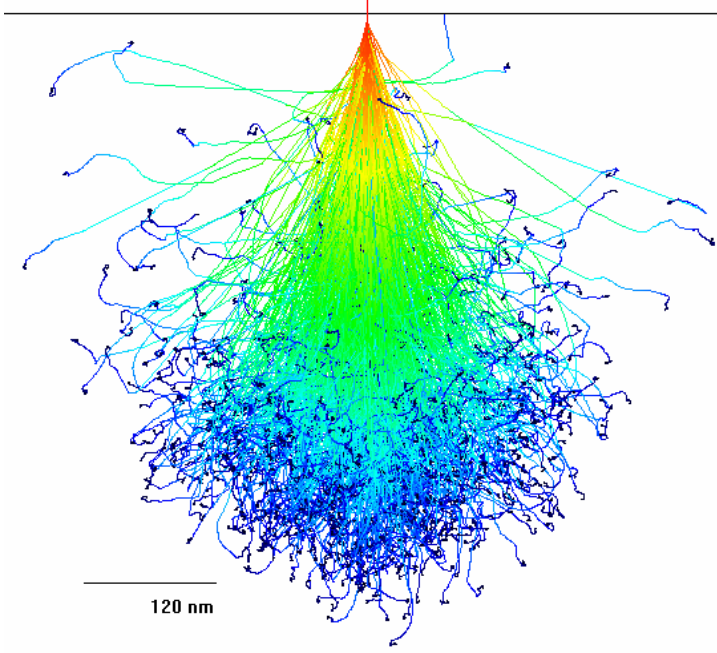

(c)

FIG. 6: Comparison of interactions (a) for a $1 \mathrm{keV}$ electron beam in carbon, (b) a $1 \mathrm{kV}$ helium ion beam in carbon, and (c) a $30 \mathrm{kV}$ helium ion beam in carbon.

energy of $10 \mathrm{keV}$ the average time between electron entry and subsequent backscattering varies from 20 femptoseconds $\left(2 \times 10^{-14} \mathrm{sec}\right)$ for carbon to about 2 femptoseconds $\left(2 \times 10^{-15} \mathrm{sec}\right)$ for gold. This timing varies with 
the energy and hence the velocity of the incident electron, so that at $30 \mathrm{keV}$ the corresponding times for carbon and gold would be 80 f.secs and 8 f.secs, respectively. As shown in Figure 5 the "splash" profiles which plot the fraction of BSE signal emerging after a given time interval have shapes which vary systematically with the atomic number of the target and transit times which show considerable variation in their magnitude. An experimental measurement of the transit time spectra with a precision of a few fempto-seconds would therefore provide a route to materials analysis. The main technological problems to using this approach is the difficulty of switching electron beams sufficient rapidly to provide accurate timing, and the inherent broadening in the time spectrum that results from the spread in drift times of electrons to the detector.

Both secondary electron emission and time of transit spectroscopy offer the promise of excellent efficiency, and hence speed, because the yield of SE and BSE events is high (typically $1.5 \mathrm{SE} /$ incident electron, and 0.3 $\mathrm{BSE} /$ incident electron, at low energies) as compared to that of X-rays or Auger electrons which is of the order of $10^{-6}$ events/incident electron $[7,14]$ and a much higher fraction of the emitted signals can also be collected. Analyses performed in a time measured in milliseconds rather than in minutes might thus be possible. While both SE spectroscopy and Time of Transit spectroscopy are still in the concept stage they have the potential to provide a unique analytical power to the LVSEM.

\section{DISRUPTIVE TECHNOLOGY}

If the advances outlined above are insufficient to meet the demand for performance, or if they arrive too late to meet expectations, then the disruptive technology of high performance ion beam microscopes might represent a serious challenge to the existing technology. Focused ion beam (FIB) systems are in widespread use for the micromachining of materials, but while their imaging abilities are admittedly useful they are not usually regarded as a potential high resolution device. Because the wavelength of ions is very much smaller than that of electrons accelerated to the same voltage diffraction limiting of the probe is of negligible importance. The problem comes from the relatively large source size and low brightness of most practical ion sources, In addition, conventional ion sources have a wide energy spread (because of their elevated operating temperature) and the ions generated exhibit multiple charge states, consequently chromatic aberrations dominate the optical performance especially at low voltages. A recent development [15] is the development of a nano- ion source. In this device a tungsten emitter held at cryogenic temperatures condenses helium gas to form a solid helium tip which is only a few ions in size. After alignment to place one of these helium atoms on axis a field is applied and the resultant ion beam can be extracted down the column. This source is claimed to offer a reduced brightness significantly higher than that of a nano-tip electron emitter operating at the same voltage, a low chromatic energy spread, and a sub-nanometer source size. When mated with conventional ion optics such a system could be expected to generate an ion beam of nanometer size containing a current of $0.1 \mathrm{nA}$ or more.

Ion beams have many advantages over electron beams. At low and medium accelerating voltages the penetration of most ion beams is comparable with and usually significant lower than that of an electron beam (Figure 6 ) ensuring good surface information. In addition ion beams have a high SE yield, a wide variation of yield with sample chemistry, and display strong channeling effects which can lead to valuable crystallographic contrast in an image. At low voltages $(<5 \mathrm{kV})$ there is also significant ion backscattering which may result in additional image information. Both X-ray photons [16] and secondary ions are excited by the beam so some intriguing microanalytical modes might now also be possible. Possible drawbacks to be considered include positive surface charging at most energies which could reduce the secondary electron yield, sputtering damage of the sample surface, and the implantation of the incident ions, although at realistic imaging currents these effects may not be significant.

\section{SUMMARY}

The SEM, and especially the low voltage SEM, have become the most widely used electron beam tools because of their imaging performance and versatility. The advances discussed in the paper indicate that still further improvements in performance can be anticipated, positioning the LVSEM to remain the microscope of choice for emerging field in nanotechnology.

\section{Acknowledgments}

The author is grateful to Drs. Dale Newbury (NIST), Scott Davilla (4Pi Inc.), Charlie Nielson (JEOL USA), and Clarke Fenner (ALIS Corp.) for helpful discussions. Portions of this work were supported by the International SEMATECH Manufacturing Initiative (ISMI).
[1] O. L. Scherzer, Zeitchrift Physik 101, 593 (1936).

[2] O. L. Scherzer, Optik2,114 (1947).

[3] J. Zach and M. Haider, Nucl. Instrum. and Methods in Physics Research (Section A), 363, 316 (1995).

[4] O. L. Krivanek, N. Delby, and A. R. Lupini, Ultramicroscopy 78, 161 (1999).

[5] K. Honda and S. Takashima, JEOL News 38, 36 (2003).

[6] J. Goldstein, D. E. Newbury, D. C. Joy, C. Lyman, P. E. Echlin, E. Lifshin, L. Sawyer, and J. Michael, Scan- ning Electron Microscopy and X-ray Microanalysis, 3rd Ed. 2nd printing, (New York,: Kluwer, 2005).

[7] A database of backscatter and secondary electron yields is available at http://pciserver.bio.utk.edu/metrology.

[8] J. Spence, W. Qian, and M. Silverman, J. Vac. Sci. Tech. A 12, 542 (1994).

[9] Z. Radi, A. Vladar, and M. Postek, SCANNING, in press (2005).

[10] D. C. Joy, N. Khanna, and D. Braski, Proc. of SPIE, 
Metrology, Inspection, and Process Control for Microlithography XIII, 3677, 621 (1996).

[11] D. C. Joy, M. S. Prasad and H. H. Meyer, J. Microscopy 215, 77 (2004).

[12] D. C. Joy, Microscopy and Microanalysis 11, (Suppl. 2), 190, (2005).

[13] D. C. Joy, Monte Carlo Modeling for Electron Microscopy and Microanalysis, (Oxford University Press, New York,
1995).

[14] M. S. Prasad and D. C. Joy, SCANNING 25, 210 (2003).

[15] ALIS Corporation: Peabody, MA (www.aliscorporation.com). For a recent press release see http://www.eetimes.com/showArticle.jhtml?articleID= 165700941.

[16] L. Giannuzzi, SCANNING 27, 165 (2005). 